Research Article

\title{
The Relationship between Resilience and Academic Burnout among Nursing and Midwifery Students in Kermanshah, Iran
}

\author{
Maryam Janatolmakan, ${ }^{1}$ Yousef Torabi, ${ }^{2}$ Shahab Rezaeian $\mathbb{D}^{1},{ }^{1}$ Bahare Andayeshgar $\mathbb{D},{ }^{3}$ \\ Amirabbas Dabiry, ${ }^{2}$ and Alireza Khatony $\circledast^{4,5}$ \\ ${ }^{1}$ Clinical Research Development Center, Imam Reza Hospital, Kermanshah University of Medical Sciences, Kermanshah, Iran \\ ${ }^{2}$ Student Research Committee, Kermanshah University of Medical Sciences, Kermanshah, Iran \\ ${ }^{3}$ School of Health, Kermanshah University of Medical Sciences, Kermanshah, Iran \\ ${ }^{4}$ Social Development and Health Promotion Research Center, Health Institute, Kermanshah University of Medical Sciences, \\ Kermanshah, Iran \\ ${ }^{5}$ Infectious Diseases Research Center, Kermanshah University of Medical Sciences, Kermanshah, Iran
}

Correspondence should be addressed to Alireza Khatony; akhatony@gmail.com

Received 26 December 2020; Revised 4 July 2021; Accepted 12 July 2021; Published 21 July 2021

Academic Editor: Ehsan Namaziandost

Copyright $\odot 2021$ Maryam Janatolmakan et al. This is an open access article distributed under the Creative Commons Attribution License, which permits unrestricted use, distribution, and reproduction in any medium, provided the original work is properly cited.

\begin{abstract}
Background. Nursing and midwifery students have relatively high levels of academic burnout. One of the mechanisms to combat this issue is resilience. The results related to the association between academic burnout and resilience indicate a negative association, but various studies have reported different correlation coefficients. Therefore, the current study was aimed to investigate the association between resilience and academic burnout among nursing and midwifery students. Methods. A total of 240 nursing and midwifery students were recruited in this cross-sectional study using stratified random sampling. Data were collected by a demographic information questionnaire, the Connor-Davidson Resilience Scale (CD-RISC), and the Maslach Burnout Inventory-Student Survey (MBI-SS). Data were analyzed by SPSS-16 using t-test, ANOVA, Spearman's correlation coefficient, and linear regression analysis. Results. The mean scores of academic burnout for nursing and midwifery students were $41.4 \pm 14.8$ and $41.2 \pm 12.3$, respectively, but the difference was not statistically significant $(p=0.368)$. The mean scores of resilience for nursing and midwifery students were $58.1 \pm 13.3$ and $52.9 \pm 13.9$, respectively, which showed a statistically significant difference $(p=0.004)$. Resilience was significantly inversely correlated with academic burnout in nursing and midwifery students $(r=-0.04, p<0.001 ; r=-0.39, p<0.001)$. Increased resilience in students decreased academic burnout $(p<0.001)$. Conclusion. Academic burnout was moderate in nursing and midwifery students, but resilience was relatively high. Given the negative correlation between resilience and academic burnout, it is necessary to strengthen resilience skills and reduce factors that cause academic burnout.
\end{abstract}

\section{Introduction}

Individuals working in any profession can be exposed to stress at the workplace. However, the nature of some occupations, including nursing and midwifery, requires that their employees be more exposed to job stress [1-6]. The teaching/ learning process, especially in the fields of nursing and midwifery, is a stressful experience. In addition to academic stress, students in these fields face various stressors such as observing patient death, fear of making mistakes at the patient's bedside, dealing with various infectious diseases, and the need to learn various skills for different roles $[7,8]$.

Prolonged exposure to emotional and interpersonal stressors may cause emotional exhaustion, skepticism, and procrastination, all of which are referred to as educational burnout syndrome [9-15]. Evidence suggests that nursing and midwifery students have relatively high levels of burnout $[5-7,16]$. In this regard, Aghajani et al., Behrozi et al., and 
Marzooghi et al. reported that nursing students in Iran experience moderate to high levels of academic burnout $[7,16,17]$. Further, Eaves and Payne reported a high rate of academic burnout in midwifery students [8].

Academic burnout may lead to unprofessional performance, academic failure, and reduced life satisfaction. Given these consequences, coping with this dilemma is of crucial importance $[8,10,12-14,18-22]$. One of the mechanisms to combat academic burnout is resilience [10, 23-25]. Resilience can be defined as the ability to overcome difficulties and turmoil and become stronger in the face of unpleasant experiences [26, 27]. Resilience can protect people against stressors and lead to success in work and life [11,26].

Various studies have investigated the association between academic burnout and resilience. Some other studies have shown a moderate to high rate of academic burnout $[28,29]$. In terms of resilience, some studies have indicated high levels of resilience [10, 24, 30,31], and some have reported low levels [32]. The results related to the association between academic burnout and resilience in nursing and midwifery students indicate a negative and significant association, but various studies have reported different correlation coefficients [10,29, 33-42].

Given the severe consequences of academic burnout and nursing and midwifery students' lack of knowledge about academic burnout and resilience, the current study was aimed to investigate the association between resilience and academic burnout and compare nursing with midwifery students. This study sought to answer the following questions: (1) what is the rate of resilience and academic burnout in nursing and midwifery students? and (2) what is the relationship between resilience and academic burnout in nursing and midwifery students?

\section{Materials and Methods}

2.1. Study Design. This cross-sectional study was conducted at Kermanshah University of Medical Sciences (KUMS) from September to December 2018.

2.2. Sample and Sampling Method. The study population included all nursing and midwifery students of KUMS in 2019. According to the results of the study conducted by Kamalpour et al. [34] and using the correlational sample size formula, with $95 \%$ confidence interval and $80 \%$ study power, the sample size was calculated to be 120 subjects. Since both nursing and midwifery students were recruited, a total of 240 participants were selected. The inclusion criteria were willingness to participate in the study, being a nursing or midwifery student, having at least one academic year, no history of psychiatric illness (according to participants' self-report), not being in the examination period, not using psychiatric drugs (according to participants' self-report), and not having thyroid (according to participants' self-report). In this study, there were no missing data, and the response rate was $100 \%$.

2.3. Study Instruments. Data were collected using a demographic information form, the Connor-Davidson Resilience
A total of 240 nursing and midwifery students were selected using the systematic sampling technique

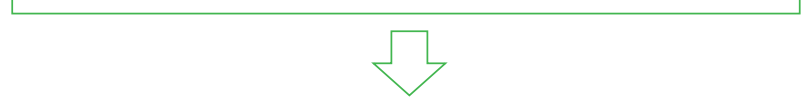

The objectives of the study were explained to the students, and if they agreed, the questionnaires were given to them

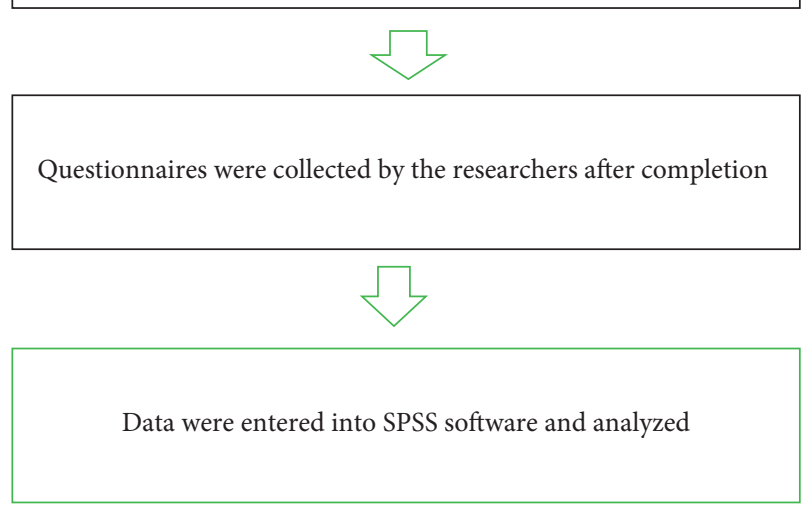

FIgURE 1: Study flow diagram.

Scale (CD-RISC), and the Maslach Burnout InventoryStudent Survey (MBI-SS). The demographic information form included five questions about the field of study, age, gender, semester, and residence. The CD-RISC was used to measure resilience [43]. Various studies have investigated the validity and reliability of these tools. Ramirez-Granizo et al. reported Cronbach's alpha of 0.86 for this tool [24].

The Persian version of CD-RISC has been psychometrically evaluated in Iran, with Cronbach's alpha levels of 0.87 and $0.89[25,26]$. Mohammadi et al. reported Cronbach's alpha level of 0.89 [22]. In the present study, the internal consistency of CD-RISC was evaluated and confirmed with Cronbach's alpha of 0.85 . The CD-RISC contains 25 questions and 4 subscales: achievement motivation (items 13, 14, 15,19 , and 25), self-confidence (items $3,5,10,12,16,17,20$, 22 , and 23), tenacity (items $4,6,18$, and 21 ), and adaptability (items 1, 2, 7, 8, 9, 11, and 24). A 5-point Likert scale is used to answer the questions: "not true at all" (0), "rarely true" (1), "sometimes true" (2), "often true" (3), and "almost always true" (4). The level of resilience is equal to the sum of the scores of all items. The total score ranges from 0 to 100 . A score range of 0 to 50 is equivalent to "low resilience" and a score range of 51 to 100 is equivalent to "high resilience."

The MBI-SS, developed by Maslach and Jackson, was used to measure academic burnout [18]. The validity and reliability of this tool have been confirmed in previous studies. Yavuz et al. examined the internal consistency of the instrument using Cronbach's alpha, and the alpha coefficients for the exhaustion, cynicism, and reduced efficacy subscales were $0.838,0.844$, and 0.875 , respectively $[15,20]$. The Persian version of MBI-SS has also been psychometrically evaluated in Iran, with alpha coefficients of $0.89,0.84$, and 0.67 for exhaustion, cynicism, and reduced efficacy 
subscales, respectively [19]. In the current study, the internal consistency of MBI-SS was evaluated and confirmed by Cronbach's alpha. The alpha coefficients for the exhaustion, cynicism, and reduced efficacy subscales were $0.85,0.80$, and 0.70 , respectively. The MBI-SS contains 15 questions, including questions $1-5,6-9$, and $10-15$, which are related to exhaustion, cynicism, and reduced efficacy subscales, respectively. Questions 10-15 score in reverse. A 7-point Likert scale is used to answer the questions: "never" (0), "very rarely" (1), "rarely" (2), "sometimes" (3), "often" (4), "more often" (5), and "always" (6). To determine the level of academic burnout, the scores of all items were added. The total score ranges of 0-30, 31-60, and 61-90 indicate mild, moderate, and severe burnout, respectively.

2.4. Data Collection Method. After obtaining the approval of the ethics committee, the list of nursing and midwifery students was obtained from the school of nursing and midwifery. A total of 240 students were selected using the systematic sampling technique. Then, based on the students' class schedule, the researcher referred to their classes to explain the study objectives to them, and if they agreed, the questionnaires were given to them to complete. To increase the accuracy of the study, the students were provided with sufficient time to fill out the questionnaires (Figure 1).

2.5. Statistical Analysis. SPSS-16 software was used to analyze and describe the data. Initially, the Kolmogorov-Smirnov test was used to check the normality of quantitative variables (i.e., age, resilience, burnout, and their components). The results showed a normal distribution for age, components of resilience, and burnout. The independent $\mathrm{t}$-test was used to compare normal quantitative variables (i.e., resilience, burnout, and emotional exhaustion components) in nursing and midwifery students. The chisquare test was used to compare nominal qualitative variables (gender and occupation) in the two groups. The independent $\mathrm{t}$-test was used to compare resilience and academic burnout between men and women as well as between students who were living in the dormitory and those who were not living in the dormitory. ANOVA test was used to compare resilience and academic burnout in students with various age ranges and academic semesters. Spearman's correlation coefficient was used to assess the association between resilience and academic burnout. Linear regression was used to investigate the factors affecting academic burnout. The significance level was set at $p<0.05$.

2.6. Ethical Considerations. The ethics committee of Kermanshah University of Medical Sciences confirmed the study. Written informed consent was obtained from all participants, and confidentiality of information was assured.

\section{Results}

The mean age of nursing students was $22.3 \pm 2.6$ years; $49.2 \%$ $(n=59)$ of them were female; and $42.5 \%(n=51)$ lived in the
TABle 1: Demographic characteristics of participants $(N=240)$.

\begin{tabular}{lccc}
\hline \multicolumn{1}{c}{ Variables } & & Nursing & Midwifery \\
\hline Age $($ mean \pm SD) & & $22.3 \pm 2.4$ & $21.6 \pm 1.7$ \\
\hline \multirow{2}{*}{ Gender, $n(\%)$} & Male & $61(50.8)$ & - \\
& Female & $59(49.2)$ & $120(100.0)$ \\
\hline \multirow{2}{*}{ Educational } & 3 and 4 & $40(33.3)$ & $60(50.0)$ \\
semester, $n(\%)$ & 5 and 6 & $40(33.3)$ & $30(25.0)$ \\
& 7 and 8 & $40(33.3)$ & $30(25.0)$ \\
\hline \multirow{2}{*}{ Residence, $n(\%)$} & Dormitory & $51(42.5)$ & $68(56.7)$ \\
& Nondormitory & $69(57.5)$ & $52(43.3)$ \\
\hline
\end{tabular}

dormitory. The mean age of midwifery students was $21.6 \pm 1.7$ years, and $56.67 \%(n=68)$ of them were living in the dormitory (Table 1).

The mean scores of academic burnout for nursing and midwifery students were $41.4 \pm 14.8$ and $41.2 \pm 12.3$ (out of 90 ), respectively, which were not significantly different. For the academic burnout subscales (exhaustion, cynicism, and reduced efficacy), the mean scores for nursing students were $13.2 \pm 7.2,10.0 \pm 6.5$, and $18.2 \pm 6.9$, respectively. For midwifery students, the mean scores of exhaustion, cynicism, and reduced efficacy were $13.3 \pm 5.7,10.5 \pm 5.6$, and $17.4 \pm 5.9$, respectively. In terms of the academic burnout subscales, there was no significant association between nursing and midwifery students $(p<0.05)$.

According to the results, the mean resilience scores of nursing and midwifery students were $58.1 \pm 13.3$ and $52.9 \pm 13.9$ (out of 100), respectively, which was significantly different $(p=0.004)$. The mean scores of resilience subscales, including achievement motivation, self-confidence, tenacity, and adaptability, in nursing students were $11.7 \pm 3.4$, $20.0 \pm 5.4,9.3 \pm 2.6$, and $16.9 \pm 4.0$, respectively. The mean scores of achievement motivation, self-confidence, tenacity, and adaptability subscales in midwifery students were $10.6 \pm 3.3,18.7 \pm 5.3,7.9 \pm 2.8$, and $15.8 \pm 4.2$, respectively. In terms of resilience subscales, a significant association was observed between nursing and midwifery students $(p<0.05$; Table 2).

The results showed a significantly inverse correlation between resilience and academic burnout in nursing $(r=-0.04 ; p<0.001)$ and midwifery $(r=-0.39 ; p<0.001)$ students (Table 3 ).

Moreover, students' increased resilience decreased the academic burnout $(F=52.50 ; p<0.001$; Table 4$)$.

According to the multiple regression model, after adjusting the other variables (Table 5), the resilience, residence, and semester variables were significantly associated with burnout. Dormitory residents and students in the fifth and sixth semesters had significant burnout. Academic burnout decreased with an increase in resilience. However, no statistically significant relationship was found between the age and sex variables and academic burnout (Table 5).

\section{Discussion}

The current study was aimed to investigate the association between resilience and academic burnout in nursing and midwifery students. The results showed that nursing and 
TABLE 2: Comparison of resilience and academic burnout in nursing and midwifery students.

\begin{tabular}{|c|c|c|c|c|}
\hline \multicolumn{2}{|r|}{ Variables } & Nursing students (mean \pm SD) & Midwifery students (mean \pm SD) & $p$ value \\
\hline \multirow{5}{*}{ Resilience subscale } & Achievement motivation & $11.7 \pm 3.4$ & $10.6 \pm 3.3$ & 0.004 \\
\hline & Self-confidence & $20.0 \pm 5.4$ & $18.7 \pm 5.3$ & 0.032 \\
\hline & Tenacity & $9.3 \pm 2.6$ & $7.9 \pm 2.8$ & $<0.001$ \\
\hline & Adaptability & $16.9 \pm 4.0$ & $15.8 \pm 4.2$ & 0.028 \\
\hline & Resilience (total) & $58.1 \pm 13.3$ & $52.9 \pm 13.9$ & 0.004 \\
\hline \multirow{4}{*}{ Burnout subscale } & Cynicism & $10.0 \pm 6.5$ & $10.5 \pm 5.6$ & 0.373 \\
\hline & Reduced efficacy & $18.2 \pm 6.9$ & $17.4 \pm 5.9$ & 0.298 \\
\hline & Emotional fatigue & $13.2 \pm 7.2$ & $13.3 \pm 5.7$ & 0.842 \\
\hline & Burnout (total) & $41.4 \pm 14.8$ & $41.2 \pm 12.3$ & 0.368 \\
\hline
\end{tabular}

TABLE 3: Relationship between dimensions of resilience and burnout among nursing and midwifery students.

\begin{tabular}{lccccccc}
\hline \multirow{2}{*}{ Dimensions of resilience } & \multicolumn{2}{c}{ Cynicism } & \multicolumn{2}{c}{ Dimensions of burnout } \\
& & \multicolumn{2}{c}{ Reduced efficacy } & \multicolumn{2}{c}{ Exhaustion } \\
& Students & $r$ & $p$ value & $r$ & $p$ value & $r$ & $p$ value \\
\hline \multirow{2}{*}{ Achievement motivation } & Nursing & -0.13 & 0.142 & -0.33 & $<0.001$ & -0.15 \\
& Midwifery & -0.27 & 0.002 & -0.29 & 0.001 & -0.26 & 0.098 \\
& Nursing & -0.28 & 0.002 & -0.36 & $<0.001$ & -0.28 \\
\multirow{2}{*}{ Self-confidence } & Midwifery & -0.21 & 0.021 & -0.18 & 0.053 & -0.22 & 0.018 \\
\hline \multirow{2}{*}{ Tenacity } & Nursing & -0.41 & $<0.001$ & -0.28 & 0.002 & -0.27 & 0.003 \\
& Midwifery & -0.32 & $<0.001$ & -0.24 & 0.008 & -0.33 & $<0.001$ \\
\hline \multirow{2}{*}{ Adaptability } & Nursing & -0.40 & $<0.001$ & -0.28 & 0.002 & -0.30 & 0.001 \\
& Midwifery & -0.33 & $<0.001$ & -0.31 & $<0.001$ & -0.33 & $<0.001$ \\
\hline
\end{tabular}

TABLE 4: The relationship between academic burnout and resilience.

\begin{tabular}{|c|c|c|c|c|c|}
\hline \multirow{2}{*}{ Model } & \multicolumn{2}{|c|}{ Unstandardized coefficients } & \multirow{2}{*}{$\begin{array}{c}\text { Standardized coefficients } \\
\text { Beta }\end{array}$} & \multirow{2}{*}{$t$} & \multirow{2}{*}{$p$ value } \\
\hline & $B$ & Standard error & & & \\
\hline Constant & 73.35 & 2.59 & - & 28.29 & $<0.001$ \\
\hline Burnout & -0.432 & 0.06 & ${ }^{2}=0.18$ & -7.24 & $<0.001$ \\
\hline
\end{tabular}

TABLE 5: Factors related to academic burnout using the regression model.

\begin{tabular}{lcccc}
\hline & Variables & $B$ & SE & $p$ value \\
\hline \multirow{2}{*}{ Gender } & Male & Ref. & - & - \\
& Female & 1.79 & 2.3 & 0.454 \\
\hline \multirow{2}{*}{ Age } & Continuous & 0.01 & 0.4 & 0.978 \\
\hline \multirow{2}{*}{ Residence } & Nondormitory & Ref. & - & - \\
& Dormitory & 3.97 & 1.65 & 0.017 \\
\hline \multirow{2}{*}{ Semester } & 3 and 4 & Ref. & - & - \\
& 5 and 6 & 4.12 & 1.99 & 0.040 \\
& 7 and 8 & 1.11 & 2.04 & 0.588 \\
\multirow{2}{*}{ Field of study } & Nursing & Ref. & - & - \\
& Midwifery & -3.64 & 2.04 & 0.074 \\
\hline \multirow{2}{*}{ Resilience } & Continuous & -0.41 & 0.06 & $<0.001$ \\
\hline \multirow{2}{*}{} & & & &
\end{tabular}

midwifery students had an average level of academic burnout. Some studies have reported an average level of academic burnout in students, which is in line with the results of the present study $[9,11,21,34]$. Problems such as the high volume of lessons, multiple assignments, concerns about the future career, and financial problems are common during college education, which may consequently cause depression $[5,17,44]$. The results showed that the level of resilience in nursing and midwifery students was moderate to high, and nursing students had a higher rate of resilience than midwifery students. Resilience is individual differences in coping with and responding to difficult situations [25]; therefore, the higher resilience of nursing students than midwifery students can be related to their individual differences.

Bahadir-Yilmaz showed low resilience in Turkish nursing and midwifery students [3]. However, there is some evidence that students have a high degree of resilience $[21,34,44]$, confirming the results of the current study. High levels of resilience, as a protective factor, can help students cope with academic problems and tensions and thus reduce their academic burnout $[4,5]$.

Decreased academic burnout, in turn, can have several positive educational outcomes, including academic achievement [6]. The results showed a statistically significant and inverse association between resilience and academic burnout in nursing and midwifery students so that individuals with higher resilience had lower levels of burnout and academic failure. Several studies also suggest a significant and inverse association between resilience and 
academic burnout $[5,23,34,39]$. Students with resilience skills can manage difficult life situations and adapt themselves to stressful situations, which increases their performance and reduces their academic burnout $[2,6,34]$.

The results showed a statistically significant relationship between semester and academic burnout so that academic burnout was significantly higher in students of fifth and sixth semesters than students of third and fourth semesters. This finding is consistent with the results of Fernández-Castillo and Fernández-Prados [42] but in contrast with the results of Shin et al. and Kamalpour et al. [34, 41]. As students progress to higher semesters, their experiences in dealing with stressful events are expected to increase, which can lead to a reduction in burnout.

In the present study, academic burnout was significantly higher in students living in dormitories than those living with their families. This finding is consistent with the results of Wang et al. and Cheng et al. [11,45] but in contrast with the results of Lee [35]. It seems that living with family can reduce many of the stresses of student life and increase their resilience.

The results showed no statistically significant relationship between academic burnout and age. This finding is in contrast with the results of Oyoo [37]. In this study, academic burnout was higher in the older students than the younger ones. As students get older, their personal experiences as well as their self-confidence are expected to increase, which plays an important role in increasing their resilience and thus reducing their academic burnout.

The results also showed no statistically significant relationship between academic burnout and gender. This finding is in line with the results of Oyoo [37]. In this study, academic burnout was higher in the male students than the female ones. Due to the nature of nursing and midwifery disciplines, male and female students are expected to have a high level of resilience to be able to react appropriately to different types of stressors and not suffer from academic burnout.

4.1. Limitations. This study had some limitations. First, due to the cross-sectional nature of the study, it was not possible to investigate the causal association between variables. Data were collected through self-report, which may have affected the accuracy of the data. Interventions such as workshops are recommended to prevent academic burnout and increase resilience among students.

\section{Conclusions}

Nursing and midwifery students had moderate levels of academic burnout. In terms of resilience, nursing and midwifery students had moderate to high levels of resilience, and nursing students were more resilient than midwifery students. A significant and inverse association was found between resilience and academic burnout in nursing and midwifery students. Regarding the negative correlation between resilience skills and academic burnout, face-to-face or virtual workshops are recommended to be held to enhance students' resilience skills. Further cross-sectional and longitudinal studies are also suggested to investigate the impacts of such workshops on resilience and academic burnout.

\section{Data Availability}

The identified data sets analyzed during the current study are available from the corresponding author on reasonable request.

\section{Conflicts of Interest}

The authors declare that there are no conflicts of interest.

\section{Acknowledgments}

This article was drawn from a research project (No. 97800) sponsored by the Deputy of Research and Technology of KUMS. The authors would like to express their sincere gratitude to all the nursing and midwifery students who participated in this research. The authors thank the clinical research development center of Imam Reza Hospital for their kind help.

\section{References}

[1] Z. B. Moghadam, N. Maleki, F. Rahimikian, and M. Hosseini, "Prevalence of burnout in midwives working in teaching hospitals and health centers," Payesh (Health Monitor), vol. 13, no. 3, pp. 339-346, 2014.

[2] E. A. Ajam, T. Rezaei, and A. A. Bayani, "Relationship between hope to work and academic motivation with academic burnout," Knowledge and Health, vol. 10, no. 1, pp. 44-50, 2015.

[3] E. Bahadir-Yilmaz, "Resilience levels of junior nursing and midwifery students according to their stress coping styles," Journal of Ege University Nursing Faculty, vol. 31, pp. 52-61, 2015.

[4] F. Darban, A. Balouchi, A. Narouipour, E. Safarzaei, and H. Shahdadi, "Effect of communication skills training on the burnout of nurses: a cross-sectional study," Journal of Clinical and Diagnostic Research, vol. 10, no. 4, p. IC01, 2016.

[5] M. Hojat, M. Vergare, G. Isenberg, M. Cohen, and J. Spandorfer, "Underlying construct of empathy, optimism, and burnout in medical students," International Journal of Medical Education, vol. 6, pp. 12-16, 2015.

[6] N. Rania, A. Siri, A. Bagnasco, G. Aleo, and L. Sasso, "Academic climate, well-being and academic performance in a university degree course," Journal of Nursing Management, vol. 22, no. 6, pp. 751-760, 2014.

[7] Z. Aghajari, L. Loghmani, M. Ilkhani et al., "The relationship between quality of learning experiences and academic burnout among nursing students of Shahid Beheshti University of Medical Sciences in 2015," Electronic Journal of General Medicine, vol. 15, no. 6, 2018.

[8] J. L. Eaves and N. Payne, "Resilience, stress and burnout in student midwives," Nurse Education Today, vol. 79, pp. 188193, 2019.

[9] T. Akgemci, M. T. Demirsel, and Ö Kara, "The effect of psychological resilience on employees' burnout level," Academic Journal of Interdisciplinary Studies, vol. 2, no. 11, p. 122, 2013. 
[10] F. Amini, "The relationship between resiliency and burnout in nurses," Journal of Research Development in Nursing \& Midwifery, vol. 10, no. 2, pp. 94-102, 2013.

[11] M. Wang, H. Guan, Y. Li, C. Xing, and B. Rui, "Academic burnout and professional self-concept of nursing students: A cross-sectional study," Nurse Education Today, vol. 77, pp. 27-31, 2019.

[12] C. Atalayin, M. Balkis, H. Tezel, B. Onal, and G. Kayrak, "The prevalence and consequences of burnout on a group of preclinical dental students," European Journal of Dentistry, vol. 9, no. 3, pp. 356-363, 2015.

[13] G. Bullock, L. Kraft, K. Amsden et al., "The prevalence and effect of burnout on graduate healthcare students," Canadian Medical Education Journal, vol. 8, no. 3, pp. e90-108, 2017.

[14] E. Oliva Costa, A. Santos, A. Abreu Santos, E. Melo, and T. Andrade, "Burnout Syndrome and associated factors among medical students: a cross-sectional study," Clinics, vol. 67, no. 6, pp. 573-579, 2012.

[15] W. B. Schaufeli, I. M. Martínez, A. M. Pinto, M. Salanova, and A. B. Bakker, "Burnout and engagement in university students," Journal of Cross-Cultural Psychology, vol. 33, no. 5, pp. 464-481, 2002.

[16] N. Behroozi, M. Shahani Yeylaq, and S. M. Pourseyed, "Relationship between perfectionism, perceived stress and social support with academic burnout," 2013.

[17] R. Marzooghi, M. Heidari, and E. Heidari, "The impact of educational justice on students' academic burnout in the University of Social Welfare and Rehabilitation Science, Tehran, Iran," Strides in Development of Medical Education, vol. 10, no. 3, pp. 328-334, 2013.

[18] C. Maslach and S. E. Jackson, "The measurement of experienced burnout," Journal of Organizational Behavior, vol. 2, no. 2, pp. 99-113, 1981.

[19] Z. Rostami, M. R. Abedi, W. B. Schaufeli, S. A. Ahmadi, and A. H. Sadeghi, The Psychometric Characteristics of Maslach burnout Inventory Student Survey: A Study Students, Isfahan University, Isfahan, Iran, 2014.

[20] G. Yavuz and N. Dogan, "Maslach burnout inventory-student survey (MBI-SS): a validity study," Procedia - Social and Behavioral Sciences, vol. 116, pp. 2453-2457, 2014.

[21] A. M. Beauvais, J. G. Stewart, S. DeNisco, and J. E. Beauvais, "Factors related to academic success among nursing students: A descriptive correlational research study," Nurse Education Today, vol. 34, no. 6, pp. 918-923, 2014.

[22] A. Mohammadi, M. Aghajani, and G. Zehtabvar, "Addiction and its relation with resilience and emotional components," Iranian Journal of Psychiatry and Clinical Psychology, 2011.

[23] Z. T. Kharameh, F. Sharififard, H. Asayesh, and M. R. Sepahvandi, Academic Resilience and Burnout Relationship of the Student, Qom University of Medical Sciences, Qom, Iran, 2016.

[24] I. A. Ramirez-Granizo, M. Sánchez-Zafra, F. Zurita-Ortega, P. Puertas-Molero, G. González-Valero, and J. L. UbagoJiménez, "Multidimensional self-concept depending on levels of resilience and the motivational climate directed towards sport in schoolchildren," International Journal of Environmental Research and Public Health, vol. 17, no. 2, p. 534, 2020.

[25] S. J. Seyed mahmodi, G. Rahimi, and N. Mohammadi, "Factors affecting resilience in people with psychological trauma," Clinical Psychology Research and Counseling, vol. 1, no. 1, pp. 5-14, 2012.

[26] G. McDonald, D. Jackson, L. Wilkes, and M. Vickers, "Personal resilience in nurses and midwives: effects of a work- based educational intervention," Contemporary Nurse, vol. 45, no. 1, pp. 134-143, 2013.

[27] L. J. Thomas and S. H. Revell, "Resilience in nursing students: an integrative review," Nurse Education Today, vol. 36, pp. 457-462, 2016.

[28] T. Kristanto, W. S. Chen, and Y. Y. Thoo, "Academic burnout and eating disorder among students in Monash University Malaysia," Eating Behaviors, vol. 22, pp. 96-100, 2016.

[29] S. Kutluturkan, E. Sozeri, N. Uysal, and F. Bay, "Resilience and burnout status among nurses working in oncology," Annals of General Psychiatry, vol. 15, no. 1, p. 33, 2016.

[30] J. C. Houpy, W. W. Lee, J. N. Woodruff, and A. T. Pincavage, "Medical student resilience and stressful clinical events during clinical training," Medical Education Online, vol. 22, no. 1, Article ID 1320187, 2017.

[31] F. Zhao, Y. Guo, R. Suhonen, and H. Leino-Kilpi, "Subjective well-being and its association with peer caring and resilience among nursing vs medical students: a questionnaire study," Nurse Education Today, vol. 37, pp. 108-113, 2016.

[32] M. Calo, C. Peiris, L. Chipchase, F. Blackstock, and B. Judd, "Grit, resilience and mindset in health students," The Clinical Teacher, vol. 16, no. 4, pp. 317-322, 2019.

[33] B. R. Barker, "Grit \& resilience in learners," 2017.

[34] S. Kamalpour, M. Azizzadeh-Forouzi, and B. Tirgary, "A study of the relationship between resilience and academic burnout in nursing students," Strides in Development of Medical Education, vol. 13, no. 5, pp. 476-487, 2017.

[35] E. Lee, "Effect of resilience on academic burnout of nursing students," Journal of the Korea Academia-Industrial Cooperation Society, vol. 20, no. 6, pp. 178-187, 2019.

[36] H.-J. Lee, "Effects of resilience on academic burnout of university students majoring in public health: for group with low resilience," The Journal of Korean Society for School \& Community Health Education, vol. 20, no. 1, pp. 85-97, 2019.

[37] S. A. Oyoo, "Academic resilience as a predictor of academic burnout among form four students in Homa-Bay County, Kenya," International Journal of Educational Research, vol. 6, no. 3, pp. 187-200, 2018.

[38] C. S. Rees, B. Heritage, R. Osseiran-Moisson et al., "Can we predict burnout among student nurses? An exploration of the ICWR-1 model of individual psychological resilience," Frontiers in Psychology, vol. 7, p. 1072, 2016.

[39] M. I. Ríos-Risquez, M. García-Izquierdo, E. d. 1. A. SabucoTebar, C. Carrillo-Garcia, and M. E. Martinez-Roche, "An exploratory study of the relationship between resilience, academic burnout and psychological health in nursing students," Contemporary Nurse, vol. 52, no. 4, pp. 430-439, 2016.

[40] Z. Soltani and N. Yoosefi, "Role of psychological capital in academic burnout of Students," Education Strategies in Medical Sciences, vol. 9, no. 2, pp. 156-162, 2016.

[41] S. Shin and E. Hwang, "The effects of clinical practice stress and resilience on nursing students' academic burnout," Korean Medical Education Review, vol. 22, no. 2, pp. 115-121, 2020.

[42] A. Fernández-Castillo and M. J. Fernández-Prados, "Resilience and burnout in educational science university students: developmental analysis according to progression in the career," Current Psychology, pp. 1-10, 2021.

[43] K. M. Connor and J. R. T. Davidson, "Development of a new resilience scale: The Connor-Davidson Resilience Scale (CDRISC)," Depression and Anxiety, vol. 18, no. 2, pp. 76-82, 2003. 
[44] F. Amini, "The relationship between resiliency and burnout in nurses," Journal of Research Development in Nursing and Midwifery, vol. 10, no. 2, pp. 94-102, 2013.

[45] J. Cheng, Y. Y. Zhao, J. Wang, and Y. H. Sun, "Academic burnout and depression of Chinese medical students in the pre-clinical years: the buffering hypothesis of resilience and social support," Psychology, Health \& Medicine, vol. 25, no. 9, pp. 1094-1105, 2020. 